\title{
PRODUÇÃO CIENTÍFICA SOBRE A HOSPITALIZAÇÃO DE IDOSOS: UMA PESQUISA BIBLIOGRÁFICA
}

Ana Paula Limaํ, Maria de Fátima Mantovani², Elis Martins Ulbrich³, Evana Terezinha de Castro Zavadil ${ }^{4}$

\begin{abstract}
RESUMO: Pesquisa bibliográfica que teve como objetivo conhecer a produção científica brasileira acerca da temática “hospitalização de idosos”, em periódicos da área de saúde entre os anos de 2003 e 2008. Foram incluídos artigos na íntegra disponíveis na Internet ou na Biblioteca de Ciências da Saúde da Universidade Federal do Paraná, que apresentassem os termos: idoso hospitalizado, internação hospitalar de idosos, idoso internado/atendido em hospital e assistência hospitalar a idosos, cuidados de enfermagem, enfermagem gerontológica em seu título ou como descritores; e incluíssem descrição do procedimento metodológico adotado. Foram selecionados 38 artigos, distribuídos em quatro categorias: aspectos epidemiológicos, cuidados e cuidadores, especificidades de internação e outros. Verificou-se uma maior concentração de artigos na área da saúde coletiva e a falta de um modelo prático para atendimento dos idosos no âmbito hospitalar.
\end{abstract}

PALAVRAS-CHAVE: Hospitalização; Idosos; Cuidados de enfermagem.

\section{THE SCIENTIFIC KNOWLEDGE PRODUCTION ABOUT ELDERLY PEOPLE INSTITUTIONALIZATION: A BIBLIOGRAPHIC RESEARCH}

\begin{abstract}
Bibliographic research on the production of scientific knowledge about “elderly people institutionalization” in Brazil. Health-related periodicals and journals published between 2003 and 2008 were consulted. Full text articles available on the Internet or in the Library of Health Sciences of the Federal University of Paraná containing the terms: institutionalized elder, institutionalization of elderly people, elder inpatient, inpatient care for elderly people, nursing care and gerontological nursing in the title or as descriptors, and also those containing the description of the methodological procedure adopted were included. The 38 articles were classified into the following four categories: epidemiological aspects, care and caregivers, specific of institutionalization and others were selected. We found that most articles focused on collective health and there was no practical model for inpatient care for elderly people.
\end{abstract}

KEYWORDS: Institutionalization; Aged people; Nursing care.

\section{PRODUCCIÓN CIENTÍFICA SOBRE LA HOSPITALIZACIÓN DE ANCIANOS: UNA INVESTIGACIÓN BIBLIOGRÁFICA}

RESUMEN: Investigación bibliográfica que tuvo como objetivo conocer la producción científica brasileña sobre el tema “hospitalización de ancianos”, en periódicos del área de salud entre los años de 2003 y 2008 . Fueron incluidos artículos en su integridad disponibles en Internet o en la Biblioteca de Ciencias de la Salud de la Universidad Federal del Paraná, que presentaron los términos: anciano hospitalizado, internación hospitalaria de ancianos, anciano internado/atendido en hospital y asistencia hospitalaria a ancianos, cuidados de enfermería, enfermería gerontológica en su título o como descriptores; y presentando también la descripción del procedimiento metodológico adoptado. Fueron seleccionados 38 artículos, distribuidos en cuatro categorías: aspectos epidemiológicos, cuidados y cuidadores, especificidades de internación y otros. Se verifico la mayor concentración de artículos en el área de la salud colectiva y la falta de un modelo práctico para atendimiento de los ancianos en el ambiente hospitalario.

PALABRAS CLAVE: Hospitalización; Ancianos; Atencíon en enfermaría

${ }^{1}$ Enfermeira graduada pela Universidade Federal do Paraná-UFPR.

${ }^{2}$ Doutora em Enfermagem. Professor Adjunto do Departamento de Enfermagem da UFPR. Coordenadora do Grupo de Estudos Multiprofissional em Saúde do Adulto-GEMSA-UFPR.

${ }^{3}$ Mestranda do curso de Enfermagem da UFPR. Membro do GEMSA-UFPR.

${ }^{4}$ Mestranda do curso de Enfermagem da UFPR. Membro do GEMSA-UFPR.

Autor correspondente:

Maria de Fátima Mantovani

Universidade Federal do Paraná

Rua Padre Camargo, 280 - 80060-240 - Curitiba-PR, Brasil

Recebido: 12/03/09

E-mail:mantovani@ufpr.br

Aprovado: 16/11/09

Cogitare Enferm 2009 Out/Dez; 14(4):740-7 


\section{INTRODUÇÃO}

O mundo contemporâneo tem como característica o envelhecimento populacional, impulsionado pela redução das taxas de mortalidade e melhores condições de vida na maioria dos países. Esse fenômeno mundial é bastante perceptível nos países em desenvolvimento como o Brasil, onde o número de idosos passou de três milhões em 1960, para vinte milhões em $2007^{(1)}$, os quais são definidos pela Lei ${ }^{\circ} 10.741$, de $1^{\circ}$ de outubro de 2003, como as pessoas com idade igual ou superior a 60 anos $^{(2)}$.

O envelhecimento pode ser compreendido como um processo natural, contínuo e inerente a todos os animais, que começa logo após o nascimento. Nos seres humanos, este processo é influenciado pelo ambiente físico, político e cultural no qual ele está inserido. Por isso cada indivíduo envelhece de forma distinta e particular ${ }^{(3)}$.

O rápido aumento da população idosa, observado no Brasil, resulta em uma crescente demanda por serviços de saúde e constitui um dos maiores desafios para as práticas de saúde pública, gerando impactos econômicos de grande magnitude para o país, pois na hospitalização, a média de permanência dos idosos ${ }^{(4)}$ foi de aproximadamente dois dias a mais do que a média apresentada pelas demais faixas etárias. O que gerou incremento no custo médio das internações hospitalares, o qual(5) foi maior entre idosos em relação às faixas etárias inferiores nas cinco regiões brasileiras, sendo suas causas, em sua maioria, relacionadas a doenças cardiovasculares, respiratórias e digestórias.

Frente a estas considerações, acredita-se que o conhecimento produzido nesta área possibilita conhecer a demanda de cuidados prestados ao idoso e as relações existentes entre o processo de envelhecimento e a necessidade de cuidados específicos. Diante disto, a questão que norteia este estudo é: Qual o perfil da produção científica em relação à hospitalização de idosos? Como objetivo, conhecer as características dos artigos que abordam a temática "hospitalização de idosos”, publicados em periódicos científicos da área de saúde entre os anos de 2003 e 2008.

\section{METODOLOGIA}

Trata-se de uma pesquisa bibliográfica, a qual é classificada $^{(6)}$ como uma modalidade de pesquisa exploratória. Este tipo de pesquisa tem como objetivo principal estabelecer o contato entre o pesquisador e 0 que já foi produzido a respeito do tema de escolha. É um importante ${ }^{(7)}$ instrumento na educação continuada do profissional da saúde, além de ser imprescindível às demais modalidades de pesquisa científica.

Para a realização desta pesquisa foram estabelecidas oito etapas, delimitadas com base nas recomendações de $\mathrm{Gil}^{(6)}$ : $1^{\mathrm{a}}$ escolha do tema da pesquisa; $2^{\mathrm{a}}$ levantamento bibliográfico preliminar esta etapa objetiva a familiarização do pesquisador com o tema a ser estudado. Foram realizadas leituras acerca do tema em livros, bem como um levantamento inicial no site da biblioteca virtual em saúde-BIREME, utilizando-se a pesquisa por relevância com os termos "hospitalização" e "idoso"; $3^{\text {a }}$ formulação do problema ou questão norteadora - a questão norteadora desta pesquisa foi: Qual o perfil da produção científica em relação a hospitalização de idosos?; $4^{a}$ elaboração do plano provisório de assunto - a finalidade desta etapa é definir o que se pretende investigar em cada estudo/ artigo a ser analisado. Para viabilizá-la, foi elaborado um instrumento para coleta de dados contendo: o tipo de estudo, ano de publicação, procedência, objetivos e as considerações/conclusões, a fim de facilitar a organização lógica do assunto e a análise das variáveis selecionadas; $5^{a}$ busca das fontes e seleção do material - as fontes utilizadas foram artigos científicos publicados em periódicos da área de ciências da saúde.

A busca dos artigos foi realizada na biblioteca virtual em saúde - disponível na página da BIREME - sendo que as bases de dados utilizadas foram: LILACS (Literatura Latino-Americana e do Caribe em Ciências da Saúde), SciELO (Scientific Electronic Library Online) e BDENF (Base de Dados de Enfermagem). Foi realizada uma busca pelos Descritores em Ciências da Saúde (DeCS). Foram selecionados os descritores: hospitalização, internação hospitalar, idoso, pacientes internados, enfermagem geriátrica e cuidados de enfermagem.

Durante a busca dos artigos, os descritores foram agrupados da seguinte maneira: "idoso" e "hospitalização" (245 artigos), "idoso” e "assistência hospitalar" (2 artigos), "idoso" e "pacientes internados" (144 artigos), "idoso" e "cuidados de enfermagem" (168 artigos) e "enfermagem geriátrica” e "hospitalização” (21 artigos). A utilização do descritor enfermagem geriátrica deve-se ao fato, de que, a palavra idoso é substituída pela especialidade.

Os critérios para inclusão de artigos foram: presença dos termos escolhidos no título ou presença dos descritores anteriormente citados; artigos na 
íntegra, disponíveis na Internet ou na Biblioteca de Ciências da Saúde da Universidade Federal do ParanáBCS-UFPR; artigos em português, inglês ou espanhol; período de publicação compreendido entre janeiro de 2003 e junho de 2008; estudos/pesquisas produzidos no Brasil; presença de descrição do procedimento metodológico adotado.

Mediante a leitura dos resumos dos artigos encontrados, 44 foram selecionados, contudo, quatro não foram localizados; não estavam disponíveis na internet e não pertenciam ao acervo da Biblioteca do Setor de Ciências da Saúde/UFPR. Dois dos artigos foram excluídos por não apresentarem nenhuma informação sobre a metodologia empregada, totalizando 38 artigos.

Na sexta etapa, leitura do material selecionado e aplicação do instrumento de coleta de dados, os artigos foram lidos na íntegra e, concomitantemente, foi preenchido o instrumento de coleta de dados elaborado na $4^{\text {a }}$ fase. Neste momento também as sínteses dos artigos lidos foram transferidas para as tabelas de apresentação. Na sétima etapa, organização lógica do assunto, foram agrupados os resultados e recomendações apresentadas pelos autores em quatro categorias, elaboradas com a similaridade dos assuntos estudados pelos pesquisadores, respeitando a exclusão mútua e homogeneidade: aspectos epidemiológicos na qual foram inseridos sete artigos; cuidados e cuidadores - categoria que contemplou dez artigos; especificidades de internação - que contou com 13 artigos; e outros - na qual foram enquadrados oito artigos. Na oitava etapa, redação final, reuniram-se todas as conclusões formuladas durante a realização deste trabalho.

\section{RESULTADOS E DISCUSSÃO}

Nesta pesquisa, 38 artigos atenderam aos critérios de inclusão previamente definidos e foram analisados - 13 eram da área de saúde coletiva, 13 da área de enfermagem, sete da medicina e cinco das demais áreas da saúde. Quanto ao tipo de estudo, 27 adotaram a análise quantitativa dos dados; nove possuíam cunho qualitativo e dois optaram pelo tipo de estudo quanti-qualitativo; 18 revistas científicas apareceram como fonte dos artigos selecionados.

Com relação ao local de produção das pesquisas, notou-se a grande expressividade das instituições localizadas no Estado de São Paulo, as quais produziram $55,2 \%$ (21) dos artigos que compuseram a amostra.
Apenas três artigos provieram das regiões norte e nordeste.

Quanto ao ano de publicação, os artigos distribuíram-se heterogeneamente da seguinte maneira: três em 2003, 11 em 2004, oito em 2005, quatro em 2006, 11 em 2007 e apenas um artigo no ano corrente. A busca pelos artigos deu-se até o mês de junho de 2008, fato que pode explicar o pequeno número de publicações nesse ano.

\section{Aspectos epidemiológicos}

Os estudos incluídos nesta categoria eram, exclusivamente, quantitativos. Três basearam-se nas informações obtidas no Sistema de Informações Hospitalares-SIH-SUS e recomendaram a utilização dessa fonte de dados para subsidiar ações de saúde à população idosa. Os objetivos dispostos nos artigos apresentaram os verbos: analisar, comparar, estratificar, traçar perfil, monitorar, identificar e relacionar, o que sugere uma tendência diagnóstica dos estudos.

As internações hospitalares da população idosa tiveram grande prevalência, chegando a representar um terço do total de hospitalizações e apresentando prolongados períodos de permanência, em comparação às demais faixas etárias ${ }^{(8)}$. Foi possível verificar que a maioria das internações de idosos é do sexo feminino ${ }^{(8-}$ ${ }^{12)}$ fato que se relaciona à estatística nacional na qual há o predomínio de mulheres nas faixas etárias superiores aos 60 anos $^{(1)}$.

A análise dos dias de permanência nas instituições evidenciou que as doenças dos sistemas circulatório e respiratório, as neoplasias e as doenças do sistema digestório foram os grupos de agravos com os maiores tempos de permanência, seguidos pelos transtornos mentais e comportamentais e as doenças do sistema geniturinário ${ }^{(13)}$.

As taxas de mortalidade ${ }^{(8)}$ associaram-se à idade e ao motivo de internação, especialmente às doenças infecciosas, respiratórias, os transtornos mentais e comportamentais, doenças endócrinas, as doenças do sistema circulatório e as neoplasias.

A presença de doenças associadas aumentou em aproximadamente seis vezes o risco de morte ${ }^{(10)}$, em contrapartida, a correlação entre o sexo e taxa de mortalidade não se mostrou relevante ${ }^{(8,11)}$. Fatores associados como a presença de comorbidades e 0 estado funcional determinaram piores prognósticos ${ }^{(11)}$.

Constatou-se que os idosos com doenças em estágio avançado são submetidos a menos 
procedimentos invasivos e menos intervenções onerosas quando comparados a pacientes jovens com disfunções semelhantes e que a garantia de assistência hospitalar a esta faixa etária demanda mais recursos humanos, materiais, financeiros e maior tempo de atendimento ${ }^{(13)}$.

\section{Especificidades de internação}

Dos dez artigos incluídos nesta categoria, apenas um era de natureza qualitativa. A ocorrência de infecções hospitalares, internações por doenças respiratórias, fraturas de fêmur, depressão, cardiopatias, pneumonia, condutas alimentares, delirium, tuberculose pulmonar e quedas compuseram o rol de assuntos abordados.

Nos objetivos dos artigos incluídos nessa categoria apareceram os verbos: investigar, caracterizar, analisar, avaliar, comparar, descrever, verificar e identificar. Pode-se notar, portanto, que o foco dos estudos está na descrição, na percepção das práticas assistenciais e nas análises de algumas ocorrências específicas nas internações dos idosos.

Entre elas observou-se ${ }^{(14)}$ a grande relevância das quedas de mesmo nível para a ocorrência de fraturas de fêmur em idosos, também, os casos de agressão, que representaram $1,12 \%$ das causas das fraturas. Os principais tipos de fratura foram a transtrocanteriana $(58,43 \%)$ e a de colo de fêmur (38,20\%) e o tratamento instituído em $88,76 \%$ dos pacientes foi a intervenção cirúrgica. O período médio de internação foi de 12,69 dias e a taxa de mortalidade foi de $14,61 \%$.

Verificou-se ${ }^{(15)}$ alta prevalência de recidiva de quedas entre os idosos, sendo que metade delas teve causa relacionada à inadequação do ambiente físico, seguida por doenças neurológicas e cardiovasculares. Não foi verificada relação direta entre o uso de medicação e a ocorrência de quedas.

O resultado mais comum das quedas foi a fratura, dentre elas, a mais frequente foi a de fêmur, de rádio e clavícula. Após a ocorrência de fraturas, a consequência mais citada pelos idosos foi o medo de voltar a cair. Houve aumento de dificuldade e dependência para realização das atividades da vida diária-AVD ${ }^{(15)}$.

A taxa de mortalidade relacionada às quedas foi de $28 \%$ e os óbitos decorreram de complicações como a embolia e lesões neurológicas, resultantes do trauma intenso após a queda. Muitos desses idosos permaneceram acamados, apresentaram confusão mental, pneumonia e úlcera de decúbito ${ }^{(15)}$. As quedas $^{(16)}$ afetam negativamente a qualidade de vida dos idosos e medidas preventivas simples, como a adaptação do espaço físico do domicílio, poderiam evitá-las.

Os fatores de risco associados à ocorrência de infecção hospitalar ${ }^{(17)}$ foram elencados, dentre eles estiveram a realização de colangiografia, a presença de Diabetes Mellitus, doença pulmonar obstrutiva crônica-DPOC, utilização de cateterismo vesical, a presença de infecção comunitária e o uso de ventilação mecânica. Notou-se que as topografias de maior prevalência da infecção hospitalar foram o trato respiratório, as vias urinárias e o sítio cirúrgico, em consonância com outro estudo ${ }^{(18)} \mathrm{o}$ qual apresentou como locais de infecção mais frequentes o sistema respiratório, urinário, a pele e partes moles e o sistema gastrointestinal, decrescentemente.

A incidência de depressão em idosos ${ }^{(19)}$, nos graus leve, moderado ou severo, segundo a escala de Yesavage, foi de 46\%. Essa prevalência foi considerada elevada quando comparada a outros dados encontrados na literatura ${ }^{(20)}$, os quais evidenciaram que 31\% dos sujeitos possuíam depressão.

Com relação à pneumonia em idosos ${ }^{(21)}$, observou-se que eles podem apresentar sinais e sintomas diferentes dos observados em adultos jovens e que, muitas vezes são interpretados como situações crônicas ou preexistentes, o que acaba por retardar o diagnóstico, além de aumentar os índices de mortalidade nessa população. Dentre as variáveis pesquisadas, apenas a necessidade do uso de ventilação mecânica associou-se significativamente à ocorrência de óbito, independentemente do grupo de estudo ${ }^{(22)}$.

Verificou-se no período compreendido entre os anos de 1995 e 2002, uma significativa redução de internações relacionadas às doenças respiratórias, sendo que a intervenção vacinal, iniciada em abril de 1999, pode ter sido a mais provável causa desse fato. Entretanto, em outros estudos ${ }^{(23-24)}$ não houve associação entre doença pulmonar, referida por idosos, e a vacinação contra influenza.

\section{Cuidados e Cuidadores}

Esta categoria incluiu 12 artigos, todos produzidos por enfermeiros e/ou acadêmicos de enfermagem, sendo que 11 foram publicados em periódicos específicos da profissão. Com relação ao tipo de estudo: sete artigos utilizaram a abordagem qualitativa, quatro utilizaram a abordagem quantitativa e um foi do tipo quanti-qualitativo. 
Contemplaram-se assuntos como: atividades de lazer durante a internação, participação do acompanhante familiar no cuidado, papéis desempenhados por acompanhantes e visitantes, nível de dependência dos cuidados de enfermagem, concepções de auxiliares e idosos sobre o cuidado hospitalar, exame físico do idoso, reflexões acerca do cuidado, diagnósticos e intervenções de enfermagem, necessidades de cuidados entre idosos e não-idosos, demanda de trabalho da equipe de enfermagem e aspectos éticos da prática de enfermagem.

Os verbos citados nos objetivos foram: identificar, relacionar, analisar, compreender, conhecer, verificar, refletir, comparar, descrever, propor intervenções e desenvolver um modelo teórico. As principais sugestões dos artigos referem-se a ações com a finalidade de manter a autonomia do idoso e promover a sua qualidade de vida, diminuindo, consequentemente, os índices de internação(25-26) e recomenda-se a especialização dos profissionais de enfermagem nas áreas de gerontologia e geriatria ${ }^{(26-28)}$.

Quanto às atividades de lazer ${ }^{(29)}$ praticadas pelos idosos durante o período de internação, as mais frequentes foram: conversas, visitas, caminhadas pelos corredores do hospital e a televisão. O desejo de ocupar o tempo livre para minimizar o efeito indesejável das hospitalizações foi manifestado pelos idosos, entretanto, vários fatores foram identificados como limitantes ou inibidores para isto - dificuldade ou impedimento à deambulação, baixa acuidade visual e auditiva, as restrições impostas pelo cuidado e pela área física e a baixa disponibilidade de lazer no ambiente.

Relatos de acompanhantes ${ }^{(30)}$ e profissionais de enfermagem acerca da participação do acompanhante familiar no cuidado ao idoso, verificou que o vínculo afetivo e fatores pessoais dos cuidadores como a força de vontade, a coragem e a religiosidade facilitam o cuidado. A comunicação e as orientações prestadas pela equipe de enfermagem são fundamentais para a participação do familiar no cuidado. Além disso, as ações de enfermagem são observadas pelo acompanhante, que posteriormente procura reproduzi-las.

As experiências, tanto de visitante quanto de acompanhante, são desagradáveis aos familiares nos casos de internações prolongadas ou quando o paciente não tem um bom prognóstico. Para o familiar visitante, o enfermeiro exerce o papel de dificultador do convívio com o seu ente durante a hospitalização; o visitante tenta driblar as regras impostas e, geralmente, não compreende as dificuldades do processo de trabalho da equipe de enfermagem, por não ter um contato mais próximo com a mesma ${ }^{(31-32)}$.

Em contrapartida, o familiar que desempenha o papel de acompanhante sente-se acolhido pela equipe e satisfeito com a oportunidade a ele concedida e, em geral, mostra-se solidário e compreende as dificuldades da equipe de enfermagem, além de assumir papel de cuidador. Sugere-se maior flexibilidade às regras que restringem o contato do paciente com seus familiares e a promoção de conforto aos acompanhantes. Os autores ressaltam a importância de o enfermeiro equilibrar suas funções de gerência e assistência e considerar a família como parte do cliente assistido ${ }^{(31-32)}$.

O nível de dependência dos cuidados de enfermagem pelos idosos ${ }^{(25)}$, analisado, constatou que $36,6 \%$ dos pacientes dependiam de cuidados intermediários; 33,3\% de cuidados mínimos, 26\% necessitavam de cuidados semi-intensivos e $4 \%$ de cuidados intensivos. Pôde-se notar que há necessidade de intervenções no âmbito da saúde pública, que visem à promoção do envelhecimento saudável e à consequente redução dos internamentos na população idosa.

Com relação ao cuidado prestado aos idosos hospitalizados, os profissionais relataram que o amor à profissão e a paciência levam a assistência de qualidade e os idosos responderam que a qualidade assistencial está diretamente relacionada à atenção, ao amor à profissão, à educação e à delicadeza no relacionamento interpessoal ${ }^{(26)}$.

Percebeu-se que o exame físico realizado pelos enfermeiros nos idosos hospitalizados não possui nenhum direcionamento específico, o que sugere a falta de enfoque gerontológico durante o período de formação acadêmica. A maioria dos sujeitos relatou motivação para realizar o exame físico, sendo que a principal justificativa para a não-motivação foi a sobrecarga de trabalho. Segundo os enfermeiros, o exame físico subsidia uma assistência individualizada ao paciente e enfatiza as necessidades individuais ${ }^{(27)}$.

Os diagnósticos de enfermagem mais frequentes para os idosos ${ }^{(33)}$ foram: risco para infecção, mobilidade física prejudicada, nutrição alterada ingestão menor que as necessidades corporais, déficit no autocuidado e integridade da pele prejudicada. Risco de integridade da pele, memória prejudicada e dentição prejudicada apareceram como os principais diagnósticos em outro estudo ${ }^{(34)}$.

A ideia de que os cuidados à saúde são caridade/ favores; a relação de poder exercida pelos profissionais 
pelo fato de possuírem conhecimento científico; o preconceito ao idoso e o desrespeito a sua autonomia; a necessidade de atenção por parte do idoso hospitalizado e sua vulnerabilidade durante o período de internação foram percebidos no discurso dos pacientes idosos ${ }^{(35)}$.

Apesar de a maioria dos sujeitos ter classificado a assistência de enfermagem como “ótima”, os direitos dos idosos como à privacidade, à informação e ao acompanhante não foram respeitados. Os autores reforçam a necessidade da inclusão de disciplinas sobre o envelhecimento humano durante a formação profissional da equipe de enfermagem. Ouvir o idoso no atendimento de suas necessidades configura-se como estratégia para planejar o cuidado ${ }^{(35)}$. Observouse nesta categoria uma grande variedade de assuntos relacionados à hospitalização do idoso, desde questões relativas aos acompanhantes, cuidadores e familiares até diagnósticos de enfermagem, grau de dependência e avaliação da assistência, denotando a necessidade de explorar o assunto e a grandeza do tema e do uso para o profissional de enfermagem.

\section{Outros temas abordados}

Os oito artigos incluídos nessa categoria apresentaram uma abordagem quantitativa dos dados levantados.Nutricionistas, fisioterapeutas, enfermeiros e médicos foram responsáveis pela autoria desses artigos. Dentre os assuntos abordados estão: avaliação funcional e suas alterações durante a hospitalização, associação entre fatores biomédicos e ocorrência de re-hospitalizações, avaliação nutricional e custos das hospitalizações.

Os objetivos foram: descrever, identificar, determinar, comparar e avaliar; somente um dos artigos foi publicado em revista de enfermagem os outros em revistas de saúde coletiva e medicina.

As avaliações funcionais foram abordadas por quatro artigos, que verificaram um declínio da capacidade funcional dos idosos durante o período de internação ${ }^{(36-37)}$, entretanto em outro estudo ${ }^{(38)}$, a maioria dos sujeitos apresentou melhora, provavelmente proporcionada pela assistência multidisciplinar especializada e a assistência fisioterápica existentes na unidade pesquisada. Idosos hospitalizados por motivos de urgência e cuidados paliativos permaneceram por período maior no hospital e apresentaram evolução mais significativa de sua capacidade funcional ${ }^{(38)}$.
Houve alta prevalência ${ }^{(39)}$ e alto risco para desnutrição entre os idosos hospitalizados. A diminuição da ingestão alimentar e a presença de estresse psicológico ou doença aguda, foram os principais fatores da Mini Avaliação Nutricional-MNA associados à depleção nutricional. A classificação do estado nutricional somente pelo índice de massa corporal-IMC, não se mostrou efetivo. No entanto, a adoção da MNA como método de triagem foi importante, pois apontou grupos vulneráveis - mulheres e idosos com idade igual ou superior aos 75 anos. $\mathrm{O}$ tipo de residência, escolaridade, renda, tabagismo e etilismo e as categorias do estado nutricional agrupado não influenciaram o risco para desnutrição.

Estudos $^{(39-40)}$, também apontaram elevada prevalência de desnutrição hospitalar entre os idosos. Além disso, percebeu-se pouca atenção ao estado nutricional, mesmo entre os pacientes desnutridos, revelando a necessidade de implementar avaliações e terapias nutricionais rotineiras na instituição sede da pesquisa.

Os custos das internações hospitalares de idosos foram $^{(5)}$ evidenciados nas regiões brasileiras sudeste e sul, onde apresentaram os maiores gastos em 1991, contrapondo-se à região norte, que apresentou o menor gasto. O custo médio das internações hospitalares foi maior entre idosos, quando comparado à faixa etária inferior, nas cinco regiões brasileiras.

As doenças do aparelho circulatório e respiratório consumiram cerca de metade dos custos no ano de 2001, com destaque para as doenças isquêmicas do coração. Essas doenças representaram a quinta causa mais frequente de internações hospitalares, entretanto, foram responsáveis pelo maior custo proporcional. Os resultados reforçam a necessidade de atividades de prevenção e de promoção da saúde para a redução das causas das enfermidades relacionadas aos cinco principais fatores de risco em saúde pública: hipertensão; tabagismo, consumo de álcool, dislipidemias, e obesidade/sobrepeso ${ }^{(5)}$.

\section{CONSIDERAÇÕES FINAIS}

Encontrou-se que a área de saúde coletiva é a que mais aborda a temática do envelhecimento com enfoque da epidemiologia do mesmo, entre as categorias elaboradas a que mais apresentou divergências no seu interior foi o de cuidados e cuidadores devido a grande variedade de assuntos relacionados a hospitalização do idoso. 
De uma forma geral, os estudos que atenderam os critérios de seleção concentraram-se na descrição das características dos pacientes idosos e o tratamento a eles instituído, não sendo propostos ou testados novos modelos de assistência à saúde. Ainda, muitos autores relatam que o estilo de tratamento adotado para as demais faixas etárias não tenha a mesma eficiência quando aplicado aos idosos.

Frente aos achados considera-se a necessidade da abordagem gerontológica e geriátrica durante o período de formação dos profissionais de saúde e, também, a importância da inclusão destes conteúdos nos programas de educação permanente para os profissionais de saúde, à medida que o perfil demográfico da população brasileira está em franca transição.

\section{REFERÊNCIAS}

1. Ministério do Planejamento, Orçamento e Gestão (BR). Instituto Brasileiro de Geografia e Estatística. Síntese de indicadores sociais: uma análise das condições de vida da população brasileira. IBGE, 2007. [acesso em 2008 Mai 30]. Disponível: http://www.ibge.gov.br/home/ estatistica/populacao/condicaodevida/indicadoresmini mos/sinteseindicsociais2008/default.shtm

2. Brasil. Lei $n^{0} 10.741$, de $1^{\circ}$ de outubro de 2003. Dispõe sobre o Estatuto do Idoso. Diário Oficial da República Federativa do Brasil, Brasília. [acesso em 2008 Mai 29]. Disponível: http://www.planalto.gov.br/ccivil/LEIS/ 2003/L10.741.htm.

3. Rodrigues RAP, Diogo MJD, Barros TR. O envelhecimento do ser humano. In: Rodrigues RAP, Diogo MJD, organizadores. Como cuidar dos idosos. Campinas: Papirus; 1996. p. 11-16.

4. Ministério da Saúde (BR). DataSus. Indicadores de morbidade e fatores de risco. [acesso em 2008 Mai 30]. Disponível: http://tabnet.datasus.gov.br/

5. Peixoto SV, Giatti L, Afradique ME, Lima-Costa MF. Custo das internações hospitalares entre idosos brasileiros no âmbito do Sistema Único de Saúde [periódico na Internet]. Epidemiol. Serv. Saúde. 2004 [acesso em 2008 Jun 27] 13(4):239-246. Disponível: http:/ /www.scielo.br/scielo.php?script=sci_arttext\&pid =S0103-73312008000400010

6. Gil AC. Como delinear uma pesquisa bibliográfica. $4^{\mathrm{a}}$ ed. São Paulo: Atlas; 2002.

7. Volpato ESN. Pesquisa bibliográfica em ciências biomédicas. J Pneumol. 2000;26(2):77-80.

8. Amaral ACS, Coeli CM, Costa MCE, Cardoso VS, Toledo
ALA, Fernandes CR. Perfil de morbidade e de mortalidade de pacientes idosos hospitalizados. Cad Saúde Publ. 2004 Nov/Dez;20(6):1617-26.

9. Guerra HL, Giatti L, Lima-Costa MF. Mortalidade em internações de longa duração como indicador da qualidade da assistência hospitalar ao idoso. Epidemiol Serv Saúde. 2004;13(4):247-53.

10. Iucif JRN, Rocha JSY. Estudo da desigualdade na mortalidade hospitalar pelo índice de comorbidade de Charlson. Rev Saúde Publ. 2004;38(6):780-86.

11. Feijó CAR, Bezerra ISAM, Peixoto Jr AA, Meneses FA. Morbimortalidade do idoso internado na Unidade de Terapia Intensiva de Hospital Universitário de Fortaleza [periódico na Internet]. Rev Bras Ter Intensiva. 2006 [acesso em 2008 Jun 21] 18(3). Disponível: http:// www.scielo.br/pdf/rbti/v18n3/v18n3a08.pdf

12. Maués CR, Rodrigues SMC, Cardoso HC, Cardoso HM, Freire Jr JEB, Ribeiro VC. Epidemiologia de idosos internados na enfermaria de clínica médica de um hospital público. Rev Para Méd. 2007;21(3):31-6.

13. Martin GB, Cordoni Jr L, Bastos YGL, Silva PV. Assistência hospitalar à população idosa em cidade do sul do Brasil. Epidemiol Serviços Saúde. 2006;15(1):59-65.

14. Muniz CF, Arnaut AC, Yoshida M, Trelha CS. Caracterização dos idosos com fratura de fêmur proximal em hospital escola público. Revista Espaço para a Saúde. 2007;8(2):33-8.

15. Fabrício SCC, Rodrigues RAP, Costa Jr ML. Causas e conseqüências de quedas de idosos atendidos em hospital público. Rev Saúde Publ. 2004;38(1):93-9.

16. Ribeiro AP, Souza ER, Atie S, Souza AC, Schilithz AO. A influência das quedas na qualidade de vida de idosos. [periódico na Internet]. Ciênc Saúde Col. 2008 [acesso em 2008 Set 15] 13 (4). Disponível: http:// www.scielosp.org/scielo.php?pid=S1413-81232008000 400023\&script $=$ sci_arttext

17. Villas Bôas PJF, Ruiz T. Ocorrência de infecção hospitalar em idosos internados em hospital universitário. [periódico na Internet]. Rev Saúde Publ. 2004 [acesso em 2008 Jun 13] 38 (3). Disponível: http://www.scielo.br/ $\mathrm{pdf} / \mathrm{rsp} / \mathrm{v38n3/20653.pdf}$

18. Villas Bôas PJF, Ferreira ALA. Infecção em idosos internados em instituição de longa permanência. [periódico na Internet]. Rev Assoc Med Bras. 2007 [acesso em 2008 Set 22] 53 (2). Disponível: http:// www.scielo.br/scielo.php?pid=S0104-423020070002000 16\&script=sci_abstract\&tlng=pt

19. Ferrari JF, Dalacorte RR. Uso da escala de depressão 
geriátrica de yesavage para avaliar a prevalência de depressão em idosos hospitalizados. Scientia Medica. 2007;17(1):3-8.

20. Oliveira DAAP, Gomes L, Oliveira RF. Prevalence of depression among the elderly population who frequent community centers. Rev Saúde Publ. 2006;40(4):439-44.

21. Gorzoni ML, Massaia IFDS, Fabbri RMA, Pires SL, Taddeo EF. Índice de Fine em orientações sobre pneumonia para idosos. Rev Soc Bra Clin Méd. 2007;5(2):44-8.

22. Augusto DK, Miranda LFJR, Cruz CEG, Pedroso ERP. Estudo comparativo entre pacientes idosos internados com diagnóstico clínico de pneumonia comunitária, com ou sem confirmação radiológica. [periódico na Internet]. J Bras Pneumol. 2007 [Acesso em 2008 Jun 13].33(3). Disponível: http://www.scielo.br/scielo.php? script=sci_arttext\&pid=S1806-37132007000300007.

23. Francisco PMSB, Donalisio MR, Lattorre MRDO. Internações por doenças respiratórias em idosos e a intervenção vacinal contra influenza no Estado de São Paulo. [ periódico na Internet]. Rev Bras Epidemiol. 2004 [Acesso em 2008 Jun 13]7(2). Disponível: http://www. scielo.br/scielo.php?script=sci_arttext\&pid=S1415790X2004000200011.

24. Francisco PMSB, Donalisio MR, Barros MBA, Cézar CLG, Carandina L, Goldbaum M. Fatores associados à doença pulmonar em idosos. [periódico na Internet]. Rev Saúde Publ. 2006 [acesso em 2008 Set 15]. 40(3). Disponível: http://www.scielo.br/pdf/rsp/v40n3/10.pdf.

25. Sales FM, Santos I. Profile of elderly people hospitalized and dependence levels of nursing care: identification of necessities. Texto Contexto Enferm. 2007;16(3):495-502.

26. Rodrigues MR, Brêtas ACP. As relações entre as concepções de idosos de auxiliares de enfermagem sobre o cuidado em ambiente hospitalar. Acta Paul Enf. 2003;16(4):38-48.

27. Paula JC, Cintra FA. A relevância do exame físico do idoso para a assistência de enfermagem hospitalar. Acta Paul Enferm. 2005;18(3):301-6.

28. Brum AKR, Tocantins FR, Silva TJES. O enfermeiro como instrumento de ação no cuidar do idoso. Rev Latino-Am Enferm. 2005;13(6):1019-26.

29. Jannuzzi FF, Cintra FA. Atividades de lazer em idosos durante a hospitalização. Rev Esc Enferm USP. 2006;40(2):179-87.

30. Pena SB, Diogo MJD. Fatores que favorecem a participação do acompanhante no cuidado do idoso hospitalizado. Rev Latino-Am Enferm. 2005;13(5):663-9.

31. Silva L, Bocchi SCM. A sinalização do enfermeiro entre os papéis de familiares visitantes e acompanhante de adulto e idoso. [periódico na Internet]. Rev Latino-Am Enferm. 2005[acesso em 2008 Jun 13]13(2). Disponível: http://www.scielo.br/scielo.php?pid=S0104-116920050 00200008\&script $=$ sci_arttext

32. Bocchi SCM, Silva L, Juliani CMCM, SIPRI WC. Familiares visitantes e acompanhantes de adultos e idosos hospitalizados: análise da experiência na perspectiva do processo de trabalho em enfermagem. [periódico na Internet]. Rev Latino-Am Enferm. 2007 [acesso em 2008 Jun 13] 15(2). Disponível: www.scielo.br.

33. Sakano LM, Yoshitome AY. Diagnósticos e intervenções de enfermagem em idosos hospitalizados [periódico na Internet]. Acta Paul Enferm. 2007 [acesso em 2008 Jun 13] 20(4). Disponível: http://www.scielo.br/ scielo.php?pid=S0103-21002007000400018\&script=sci _arttext.

34. Santos ASR, Souza PA, Valle AMO, Cavalcanti ACD, Sá SPC, Santana RF. Caracterização dos diagnósticos de enfermagem identificados em prontuários de idosos: um estudo retrospectivo. Texto Contexto Enferm. 2008;17(1):141-9.

35. Ferreira AMTGB, Derntl AM. Ouvindo o idoso hospitalizado: direitos envolvidos na assistência cotidiana de enfermagem. O Mundo da Saúde. 2005;29(4):510-22.

36. Siqueira AB, Cordeiro RC, Perracini MR, Ramos LR. Impacto funcional da internação hospitalar de pacientes idosos [periódico na Internet]. Rev Saúde Publ. 2004 [acesso em 2008 Set 21] 38(5):687-94. Disponível: http:/ /www.scielo.br/pdf/rsp/v38n5/21757.pdf.

37. Kawasaki K, Diogo MJDE. Impacto da hospitalização na independencia funcional do idoso em tratamento clinico. Acta Fisiatr. 2005;12(2):55-60.

38. Izzo H, Gruner T. Impacto da internação hospitalar na capacidade funcional do idoso em enfermaria geriatrica. O Mundo da Saúde. 2005;29(1):629-38.

39. Azevedo LC, Medina F, Silva AA, Campanella ELS. Prevalencia de desnutrição em um hospital geral de grande porte de Santa Catarina/Brasil. ACM Arq Catarin Med. 2006;35(4):89-96.

40. Gorzoni ML, Pires SL. Idosos asilados em hospitais gerais [periódico na Internet]. Rev Saúde Publ. 2006 [acesso em 2008 Jun 13] 40(6): 1124-1130. Disponível: http://www.scielo.br/pdf/rsp/v40n6/24.pdf. 